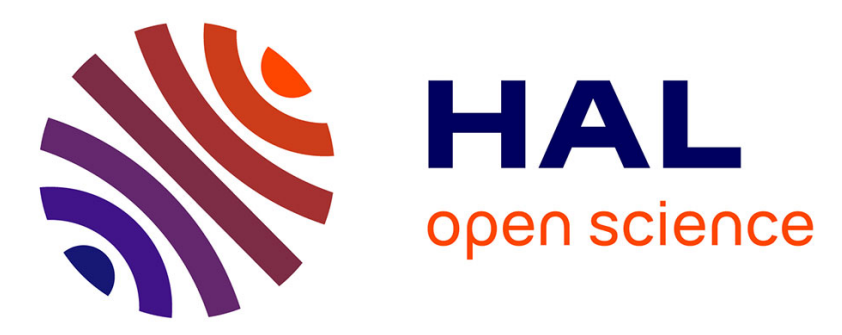

\title{
Primal-dual interior point optimization for a regularized reconstruction of NMR relaxation time distributions
}

Emilie Chouzenoux, Saïd Moussaoui, Jérôme Idier, François Mariette

\section{To cite this version:}

Emilie Chouzenoux, Saïd Moussaoui, Jérôme Idier, François Mariette. Primal-dual interior point optimization for a regularized reconstruction of NMR relaxation time distributions. IEEE International Conference on Acoustics, Speech and Signal Processing (ICASSP 2013), May 2013, Vancouver, Canada. pp.xx-xx. hal-00823603

\section{HAL Id: hal-00823603 https://hal.science/hal-00823603}

Submitted on 3 Jul 2013

HAL is a multi-disciplinary open access archive for the deposit and dissemination of scientific research documents, whether they are published or not. The documents may come from teaching and research institutions in France or abroad, or from public or private research centers.
L'archive ouverte pluridisciplinaire HAL, est destinée au dépôt et à la diffusion de documents scientifiques de niveau recherche, publiés ou non, émanant des établissements d'enseignement et de recherche français ou étrangers, des laboratoires publics ou privés. 


\title{
PRIMAL-DUAL INTERIOR POINT OPTIMIZATION FOR A REGULARIZED RECONSTRUCTION OF NMR RELAXATION TIME DISTRIBUTIONS
}

\author{
E. Chouzenoux ${ }^{1}$, S. Moussaoui ${ }^{2}$, J. Idier ${ }^{2}$ and F. Mariette ${ }^{3}$ \\ ${ }^{1}$ Université Paris-Est, \\ IGM and UMR-CNRS 8049, \\ Champs-sur-Marne, 77454 \\ Marne-la-Vallée, France \\ emilie.chouzenoux@univ-paris-est.fr \\ ${ }^{2}$ L'UNAM Université, Ecole Centrale Nantes, \\ IRCCyN and UMR-CNRS 6597, \\ 1 , rue de la Noé, BP 92101 \\ Nantes, France \\ prenom.nom@irccyn.ec-nantes.fr \\ ${ }^{3}$ CEMAGREF \\ UR TERE CS 64427, \\ 17 avenue de Cucillé \\ 35044 Rennes Cedex, \\ francois.mariette@cemagref.fr
}

\begin{abstract}
This paper deals with the reconstruction of relaxation time distributions in Nuclear Magnetic Resonance (NMR) spectroscopy. This large scale and ill-posed inverse problem is solved by the iterative minimization of a regularized objective function allowing to encode some prior assumptions on the sought distribution. The numerical optimization of the criterion is performed using a primal-dual interior point algorithm allowing to handle the non-negativity constraint. The performances of the proposed approach are illustrated through the processing of real data from a two-dimensional NMR experiment.
\end{abstract}

Index Terms - T1-T2 relaxation times, Laplace transform inversion, interior-point, primal-dual, preconditioning.

\section{INTRODUCTION}

Nuclear magnetic resonance (NMR) spectroscopy is a measurement technique allowing to determine the molecular structure and dynamics of a material. The NMR experiment consists in analyzing the relaxation process which corresponds to the re-establishment of the nuclear spin into its equilibrium state, after the application of a short magnetic pulse parameterized with a predefined flip angle $\Phi$. This process is decomposed into longitudinal and transversal dynamics, characterized by distributions of longitudinal, $T_{1}$ and/or transversal, $T_{2}$ relaxation times [1].

The reconstruction of a relaxation time distribution $\left(T_{1}\right.$, $T_{2}$ or $\left.T_{1}-T_{2}\right)$ corresponds to a numerical inversion of a Laplace transform, which is known to be an ill-posed inverse problem [2]. Moreover, such a distribution should satisfy the non-negativity constraint. In the context of NMR, two inversion strategies arise in the literature, each of them based on a specific criterion formulation and optimization algorithm. The method from [3] adopts the algorithm of [4] to minimize a Tikhonov-like criterion over the positive orthant. The size of the problem is artificially reduced by mean of a pre-processing compression step on the data. In [5], we proposed a truncated Newton optimization scheme with the ability to treat the full acquired data. The positivity constraint is implicitly handled through the use of a maximum entropy penalization criterion. The main purpose of the present paper is to propose a more general method, based on the use of an original primal-dual interior point optimization method, allowing to solve this large-scale inverse problem for any convex and differentiable regularized objective function.

The rest of this paper is organized as follows: The $T_{1}$, $T_{2}$ and $T_{1}-T_{2}$ acquisition models are presented in Section 2. Then, Section 3 presents our reconstruction method and a strategy to reduce its computational cost in the case of $T_{1}$ $T_{2}$ reconstruction. Section 4 illustrates the efficiency of the proposed scheme through synthetic and real data examples. Finally, some conclusions are drawn in Section 5.

\section{ACQUISITION MODEL}

\subsection{One-dimensional NMR acquisition}

Classical NMR experiments analyze the spin relaxation process independently, either in terms of longitudinal or transverse relaxation, leading to one dimensional (1D) measurements $x_{1}\left(\tau_{1}\right)$ or $x_{2}\left(\tau_{2}\right)$. These $1 \mathrm{D}$ NMR data are related to continuous distributions $s_{1}\left(T_{1}\right)$ and $s_{2}\left(T_{2}\right)$, also called $T_{1}$ and $T_{2}$ spectra, according to $1 \mathrm{D}$ Fredholm integrals of the first kind [6]

$$
x_{i}\left(\tau_{i}\right)=\iint k_{i}\left(\tau_{i}, T_{i}\right) s_{i}\left(T_{i}\right) d T_{i}, \quad i=1,2,
$$

where $k_{1}$ and $k_{2}$ are kernels modeling the longitudinal and transverse relaxations

$$
\begin{aligned}
& k_{1}\left(\tau_{1}, T_{1}\right)=1-\gamma e^{-\tau_{1} / T_{1}}, \\
& k_{2}\left(\tau_{2}, T_{2}\right)=e^{-\tau_{2} / T_{2}}
\end{aligned}
$$

with $\gamma$ related to the flip angle according to $\gamma=1-\cos \Phi$.

After sampling $\tau_{i}$ (resp. $T_{i}$ ) at $m_{i}$ (resp. $N_{i}$ ) discrete values, (1) reads $\boldsymbol{x}_{i}=\boldsymbol{K}_{i} \boldsymbol{s}_{i}, i=1,2$, where $\boldsymbol{y}_{i} \in \mathbb{R}^{m_{i}}, \boldsymbol{K}_{i} \in$ $\mathbb{R}^{m_{i} \times N_{i}}$ and $\boldsymbol{s}_{i} \in \mathbb{R}^{N_{i}}$. 1D NMR reconstruction amounts to the estimation of a positive spectrum $\boldsymbol{s}_{i}$ given $\boldsymbol{y}_{i}=\boldsymbol{x}_{i}+\boldsymbol{e}_{i}$, with $\boldsymbol{e}_{i}$ a noise term assumed white Gaussian, for $i=1$ (i.e., $T_{1}$ reconstruction) or $i=2$ (i.e., $T_{2}$ reconstruction). 


\subsection{Two-dimensional NMR acquisition}

Joint measurements with respect to the two relaxation parameters allow to build the two-dimensional (2D) $S\left(T_{1}, T_{2}\right)$ distribution [7], from which the one dimensional distributions can be deduced by $s_{i}\left(T_{i}\right)=\int S\left(T_{1}, T_{2}\right) d T_{j \neq i}, \quad i=1,2$.

The measured NMR data $X\left(\tau_{1}, \tau_{2}\right)$ are then related to the $T_{1}-T_{2}$ spectrum $S\left(T_{1}, T_{2}\right)$, according to a $2 \mathrm{D}$ Fredholm integral of the first kind

$$
X\left(\tau_{1}, \tau_{2}\right)=\iint k_{1}\left(\tau_{1}, T_{1}\right) S\left(T_{1}, T_{2}\right) k_{2}\left(\tau_{2}, T_{2}\right) d T_{1} d T_{2} .
$$

Note that $1 \mathrm{D}$ acquisitions can be deduced from $X\left(\tau_{1}, \tau_{2}\right)$ according to the asymptotic relations:

$$
x_{1}\left(\tau_{1}\right)=X\left(\tau_{1}, \tau_{2} \rightarrow 0\right), x_{2}\left(\tau_{2}\right)=X\left(\tau_{1} \rightarrow+\infty, \tau_{2}\right) .
$$

Experimental data consist of a series of discrete noisy samples $\boldsymbol{Y} \in \mathbb{R}^{m_{1} \times m_{2}}$ modeled by $\boldsymbol{Y}=\boldsymbol{K}_{1} \boldsymbol{S} \boldsymbol{K}_{2}^{\mathrm{t}}+\boldsymbol{E}$ with $\boldsymbol{S} \in$ $\mathbb{R}^{N_{1} \times N_{2}}$ and $\boldsymbol{E}$ a noise term assumed white Gaussian. $T_{1}-T_{2}$ NMR reconstruction aims at estimating $\boldsymbol{S}$ given $\boldsymbol{Y}$.

\section{PROPOSED RECONSTRUCTION APPROACH}

$\boldsymbol{K}_{1}$ and $\boldsymbol{K}_{2}$ are rank-deficient and very badly conditioned matrices [4]. Therefore, direct inversion is numerically unstable in both 1D and 2D cases and regularized solutions must be sought instead. In this paper, we propose a reconstruction method based on a constrained and penalized least-squares approach. The relaxation time distribution estimate is defined as the solution of

$$
\min _{\boldsymbol{s} \in \mathbb{R}^{N+}}\left(F(\boldsymbol{s})=\frac{1}{2}\|\boldsymbol{K} \boldsymbol{s}-\boldsymbol{y}\|_{2}^{2}+\beta R(\boldsymbol{s})\right),
$$

where $s:=\boldsymbol{s}_{i}, \boldsymbol{y}:=\boldsymbol{y}_{i}, \boldsymbol{K}:=\boldsymbol{K}_{i}, N:=N_{i}, i=1$ or 2 , for the 1D model, and $\boldsymbol{s}:=\operatorname{vect}[\boldsymbol{S}], \boldsymbol{y}:=\operatorname{vect}[\boldsymbol{Y}]$, $\boldsymbol{K}:=\boldsymbol{K}_{1} \otimes \boldsymbol{K}_{2}, N:=N_{1} N_{2}$, for the 2D model. The operator vect [.] corresponds to lexicographically reordering matrix elements into a vector and $\otimes$ denotes the Kronecker product. The penalization term $R(\cdot)$, whose weight is controlled by the positive parameter $\beta$, is assumed differentiable and convex. In order to handle the non-negativity constraint, an interior point algorithm based on a primal-dual approach is adopted for the resolution of (5).

\subsection{Primal-dual interior point optimization}

The primal-dual approach consists in jointly estimating $s$, and the corresponding Lagrange multipliers $\boldsymbol{\lambda}$ through the resolution of a sequence of optimization problems obtained from perturbed versions of the Karush-Kuhn-Tucker (KKT) optimality conditions:

$$
\nabla F(s)-\boldsymbol{\lambda}=\mathbf{0}, \quad \boldsymbol{\Lambda} s=\boldsymbol{\mu}_{k}, \quad(s, \boldsymbol{\lambda}) \succeq \mathbf{0},
$$

where $\boldsymbol{\Lambda}=\operatorname{Diag}(\boldsymbol{\lambda})$ and $\boldsymbol{\mu}_{k}=\mu_{k} \mathbf{1}, k \in \mathbb{N}$, is a sequence of perturbation parameters converging to 0 when $k$ tends to $+\infty$.
At each iteration $k$ of the primal-dual algorithm, an approximate solution $\left(\boldsymbol{s}_{k+1}, \boldsymbol{\lambda}_{k+1}\right)$ of (6) is calculated from one step of a Newton algorithm coupled with a linesearch strategy [8, Chap.11], according to:

$$
\left(\boldsymbol{s}_{k+1}, \boldsymbol{\lambda}_{k+1}\right)=\left(\boldsymbol{s}_{k}+\alpha_{k} \boldsymbol{d}_{k}^{s}, \boldsymbol{\lambda}_{k}+\alpha_{k} \boldsymbol{d}_{k}^{\lambda}\right)
$$

The perturbation parameter $\mu_{k+1}$ is then updated in order to ensure the algorithm convergence.

\section{Primal-dual directions}

The directions $\left(\boldsymbol{d}_{k}^{s}, \boldsymbol{d}_{k}^{\lambda}\right)$ are obtained by solving,

$$
\left[\begin{array}{cc}
\nabla^{2} F\left(\boldsymbol{s}_{k}\right) & -\boldsymbol{I}^{\mathrm{t}} \\
\boldsymbol{\Lambda}_{k} \boldsymbol{I} & \operatorname{Diag}\left(\boldsymbol{s}_{k}\right)
\end{array}\right]\left[\begin{array}{l}
\boldsymbol{d}_{k}^{s} \\
\boldsymbol{d}_{k}^{\lambda}
\end{array}\right]=\left[\begin{array}{c}
\boldsymbol{\lambda}_{k}-\nabla F\left(\boldsymbol{s}_{k}\right) \\
\boldsymbol{\mu}_{k}-\boldsymbol{\Lambda}_{k} \boldsymbol{s}_{k}
\end{array}\right]
$$

where $\nabla F(\cdot)$ and $\nabla^{2} F(\cdot)$ are, respectively, the gradient and the Hessian of criterion $F(\cdot)$, given in (5), and $\boldsymbol{I}$ is the identity matrix of $\mathbb{R}^{N}$. Rather than solving directly this system, we firstly perform the variable substitution [9],

$$
\boldsymbol{d}_{k}^{\lambda}=\operatorname{Diag}\left(\boldsymbol{s}_{k}\right)^{-1}\left[\boldsymbol{\mu}_{k}-\boldsymbol{\Lambda}_{k} \boldsymbol{s}_{k}-\boldsymbol{\Lambda}_{k} \boldsymbol{d}_{k}^{s}\right],
$$

and then calculate the primal direction $\boldsymbol{d}_{k}^{s}$ from the reduced system

$$
\boldsymbol{H}_{k} \boldsymbol{d}_{k}^{s}=-\boldsymbol{g}_{k},
$$

where $\boldsymbol{H}_{k}=\nabla^{2} F\left(\boldsymbol{s}_{k}\right)+\operatorname{Diag}\left(\boldsymbol{s}_{k}\right)^{-1} \boldsymbol{\Lambda}_{k}$ and $\boldsymbol{g}_{k}=\nabla F\left(\boldsymbol{s}_{k}\right)-$ $\operatorname{Diag}\left(\boldsymbol{s}_{k}\right)^{-1} \boldsymbol{\mu}_{k}$.

\section{Computation cost reduction in the $2 \mathrm{D}$ case}

In the $2 \mathrm{D}$ reconstruction problem, matrix $\boldsymbol{K}$ is of size $m_{1} m_{2} \times N_{1} N_{2}$. Typical values are $m_{1}=50, m_{2}=5000$, $N_{1} \times N_{2}=300 \times 300$, so $\boldsymbol{K}$ and $\boldsymbol{H}_{k}$, are huge matrices whose explicit handling is almost impossible. Consequently, we propose here to overcome this difficulty by performing an approximate resolution of (10) using a preconditioned conjugate gradient (PCG). The preconditioning strategy makes use of the singular value decomposition $\boldsymbol{K}=\boldsymbol{U} \boldsymbol{\Sigma} \boldsymbol{V}^{\mathrm{t}}$. Given $\tilde{\boldsymbol{V}}$ and $\tilde{\boldsymbol{\Sigma}}$, truncated versions of $\boldsymbol{V}$ and $\boldsymbol{\Sigma}$, we define the preconditioning matrix $\boldsymbol{P}_{k}$ such that

$\boldsymbol{P}_{k}^{-1}=\tilde{\boldsymbol{V}} \tilde{\boldsymbol{\Sigma}}^{2} \tilde{\boldsymbol{V}}^{\mathrm{t}}+\beta \operatorname{Diag}\left(\operatorname{diag}\left(\nabla^{2} R(\boldsymbol{s})\right)\right)+\operatorname{Diag}\left(\boldsymbol{s}_{k}\right)^{-1} \boldsymbol{\Lambda}_{k}$.

The PCG iterations are stopped when ([10])

$$
\left\|\boldsymbol{g}_{k}+\boldsymbol{H}_{k} \boldsymbol{d}_{k}^{s}\right\| \leqslant \eta^{\mathrm{pcg}}\left\|\boldsymbol{g}_{k}\right\|
$$

Furthermore, the computation costs for calculating $F\left(s_{k}\right)$, $\nabla F\left(\boldsymbol{s}_{k}\right)$ and the products of $\boldsymbol{P}_{k}$ and $\boldsymbol{H}_{k}$ with a vector, are reduced by exploiting the factored form of the $2 \mathrm{D}$ observation model (more details can be found in [5, Sec.III.E]). 


\section{Linesearch}

The stepsize value $\alpha_{k}$ is chosen so as to ensure the convergence of the algorithm and the fulfillment of the inequalities of the pertubed KKT system (6). A common strategy is to require a sufficient decrease in a primal-dual merit function $F_{\mu}(s, \lambda)$ along both primal and dual directions. Here, we retain the merit function from [9]:

$$
F_{\mu}(\boldsymbol{s}, \boldsymbol{\lambda})=F(\boldsymbol{s})-\mu \sum_{n=1}^{N} \ln \left(\lambda_{n} s_{n}^{2}\right)+\boldsymbol{\lambda}^{\mathrm{t}} \boldsymbol{s} .
$$

Moreover, in order to handle efficiently the vertical asymptotes in $f_{\mu_{k}}(\alpha)=F_{\mu_{k}}\left(s_{k}+\alpha \boldsymbol{d}_{k}^{s}, \boldsymbol{\lambda}_{k}+\alpha \boldsymbol{d}_{k}^{\lambda}\right)$, the majorizeminimize (MM) linesearch strategy from [11], with $J$ iterations, $J \geqslant 1$, is employed to compute the stepsize.

\section{Perturbation parameter update}

The barrier parameter $\mu_{k}$ is controlled by two conditions [12]:

$$
\left\{\begin{array}{l}
\left\|\nabla F\left(\boldsymbol{s}_{k}\right)-\boldsymbol{\lambda}_{k}\right\|_{\infty} \leqslant \eta^{s} \mu_{k} \\
\left\|\boldsymbol{\Lambda}_{k} \boldsymbol{s}_{k}-\boldsymbol{\mu}_{k}\right\|_{1} / N \leqslant \eta^{\lambda} \mu_{k}
\end{array}\right.
$$

where $\eta^{s}$ and $\eta^{\lambda}$ are positive parameters. As soon as (13) is fulfilled, $\mu_{k}$ is updated using the $\mu$-criticity rule which ensures the convergence of the primal-dual algorithm [13]:

$$
\mu_{k+1}=\theta\left(\boldsymbol{\lambda}_{k}^{\mathrm{t}} \boldsymbol{s}_{k}\right) / N, \quad \theta \in(0,1) .
$$

The iterative scheme (7) is run until the fulfillment of the following condition [8, Chap.11]

$$
\mu_{k} \leqslant \mu_{\min } \text { or }\left(\left\|\nabla F\left(s_{k}\right)-\boldsymbol{\lambda}_{k}\right\|+\left\|\boldsymbol{\Lambda}_{k} \boldsymbol{s}_{k}\right\|\right) \leqslant \eta_{0} \text {. }
$$

The main steps of the resulting method are summarized in Algorithm 1, and the following settings are made:

\begin{tabular}{|c|c|c|c|c|c|c|c|}
\hline$J$ & $\theta$ & $\mu_{0}$ & $\mu_{\min }$ & $\eta^{s}$ & $\eta^{\lambda}$ & $\eta^{\mathrm{pcg}}$ & $\eta_{0}$ \\
\hline 10 & 0.5 & 1 & $10^{-8}$ & 100 & 1.9 & $10^{-3}$ & $10^{-8}$ \\
\hline
\end{tabular}

Initialize $\boldsymbol{\lambda}_{0}>\mathbf{0}$ and $\boldsymbol{s}_{0}>\mathbf{0}$

While ((15) is not satisfied) do

$$
\mid \begin{aligned}
& \text { While ((13) is not satisfied) do } \\
& \begin{array}{l}
\text { Calculate } \boldsymbol{d}_{k}^{s} \text { by solving the system (10) } \\
\text { Deduce } \boldsymbol{d}_{k}^{\lambda} \text { from (9) } \\
\text { Search } \alpha_{k} \text { by MM strategy } \\
\text { Update }\left(\boldsymbol{s}_{k+1}, \boldsymbol{\lambda}_{k+1}\right) \text { according to (7) } \\
\text { done } \\
\text { Define } \mu_{k+1} \text { according to (14). }
\end{array} \\
& \text { done }
\end{aligned}
$$

Algorithm 1: Primal-dual interior point algorithm.

\section{EXPERIMENTAL RESULTS}

We present in this section an illustration of the proposed approach through the processing of two datasets. The first one is based on simulated data whereas the second dataset is obtained from real 2D NMR measurements on an apple sample.

\subsection{Synthetic dataset}

The synthetic distribution $S^{o}\left(T_{1}, T_{2}\right)$ is a mixture of two Gaussian density functions located at $\{(0.5 s, 0.5 s),(2 s, 1.5 s)\}$ with standard deviations $\{(0.05 s, 0.05 s),(0.3 s, 0.2 s)\}$ and mixing probabilities 0.2 and 0.8 , respectively. The first density is independent while the second presents a correlation angle of $45^{\circ}$ between $T_{1}$ and $T_{2}$ variables. The $2 \mathrm{D}$ data are obtained according to the 2D observation model from Sec. 2, with $m_{1}=50, m_{2}=5000$ and $\Phi=90^{\circ}$. A white Gaussian noise is added to get a signal to noise ratio (SNR) equal to 20 $\mathrm{dB}$. We emphasize that these settings correspond to a realistic situation. The simulated data are presented in Fig. 1.

The $2 \mathrm{D}$ spectrum is reconstructed by solving (5) using the 2D model with $N_{1}=N_{2}=300$. The regularization term is chosen as $R(s)=\|s\|_{2}^{2}$ and its weight is set to $\beta=100$. Both 1D spectra result from the resolution of (5), using the $1 \mathrm{D}$ model, the $1 \mathrm{D}$ data being deduced from the $2 \mathrm{D}$ ones using (4). The same regularization strategy as in 2D is employed, with $\beta=10^{-3}$. The reconstruction algorithm is implemented
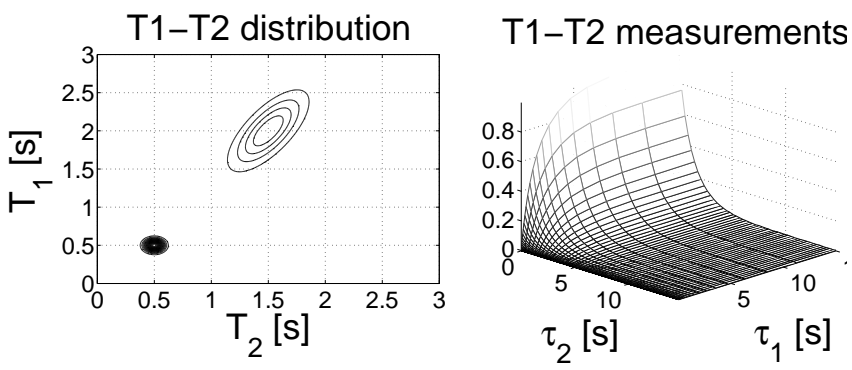

Fig. 1. Simulated $T_{1}-T_{2}$ distribution and measurements

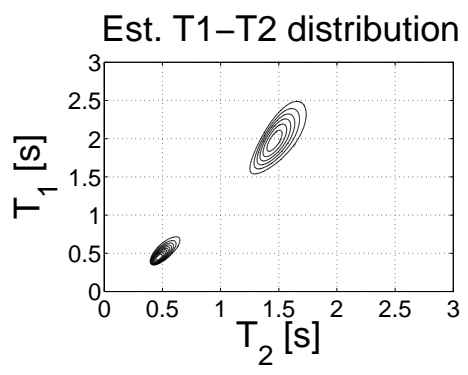

T1 and T2 distributions Est. (-) vs Ref. (--)

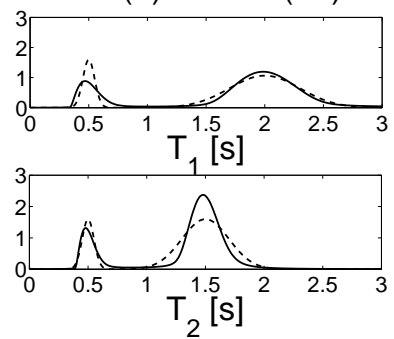

Fig. 2. Estimated $T_{1}-T_{2}, T_{1}$ and $T_{2}$ distributions using the proposed approach. The relative reconstruction errors are, respectively, $0.52,0.231$ and 0.16 . 
on Matlab 2007b and the calculations are performed using a MacbookPro having an Intel Core 2 Duo $2.4 \mathrm{GHz}$ processor and $4 \mathrm{~GB}$ of RAM (667 MHz). A positive uniform distribution is employed as an initial guess. About 20 iterations of Algorithm 1 are required to reconstruct the 1D distributions, for a computation time of about 1 second. For the 2D case, the proposed algorithm reach the stopping criterion after 36 iterations and, in spite of the large scale of the problem, the corresponding computation time is only $55 \mathrm{~s}$. Both $1 \mathrm{D}$ and 2D estimation results are shown in Fig. 2. It can be seen that the locations of both Gaussian distributions are well reconstructed. One can also observe, in Fig. 2, the similarity between the $1 \mathrm{D}$ distributions obtained by $1 \mathrm{D}$ reconstruction (straight line) and the true distributions (dashed line).

\subsection{Real dataset}

The second dataset is obtained from the analysis of a plant matter sample (apple). Measurements are made for $m_{1}=50$ values of $\tau_{1}$, non-uniformly spaced between $30 \mathrm{~ms}$ and $12 \mathrm{~s}$, and $m_{2}=10000$ echoes with a uniform time spacing of 800 $\mu \mathrm{s}$ between $600 \mu \mathrm{s}$ and $8 \mathrm{~s}$. The reconstruction is performed for $N_{1}=N_{2}=300$ values of $T_{1}$ and $T_{2}$, equally spaced between $25 \mathrm{~ms}$ and $3 \mathrm{~s}$, and a flip angle of $85^{\circ}$. Fig. 3 shows the reconstructed $2 \mathrm{D}$ spectrum for $\beta=50$, which is obtained after $260 \mathrm{~s}$ for 61 iterations of Algorithm 1. Moreover, Fig. 3 presents the $T_{1}$ (resp. $T_{2}$ ) reconstruction, obtained for $\beta=$ $10^{-3}$ (resp. $\beta=1.5$ ) after $0.3 \mathrm{~s}$ for 21 iterations (resp. $9 \mathrm{~s}$ for 35 iterations).
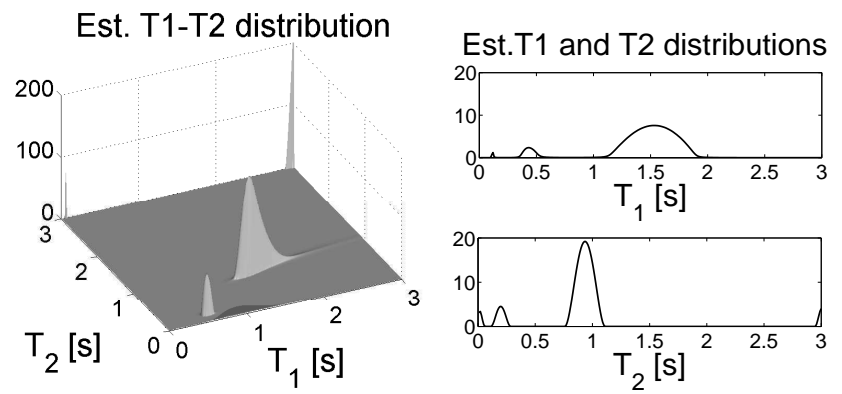

Fig. 3. Estimated distributions from the real data using the primal-dual optimization algorithm.

\section{CONCLUSION}

In this paper, we have presented a fast method for the reconstruction of NMR relaxation time distributions. The main feature of the algorithm is to use a primal-dual optimization strategy coupled with an iterative minimization in order to jointly account for the non-negativity constraint and introduce a regularization term. In the 2D case, the computation cost of the algorithm is reduced by the introduction of a preconditioned conjugate gradient and by the design of an efficient preconditioner exploiting the factorized form of the forward model. Future work will be directed at analyzing the effect of different regularization strategies on the reconstruction performances.

\section{REFERENCES}

[1] R. R. Ernst, G. Bodenhausen, and A. Wokaun, Principles of Nuclear Magnetic Resonance in One and Two Dimensions, International Series of Monographs on Chemistry. Oxford University Press, Oxford, NY, 2nd edition, 1997.

[2] E. Sternin, "Use of inverse theory algorithms in the analysis of biomembrane NMR data," in Methods in Membrane Lipids, Humana Press, Ed., vol. 400, pp. 103-125. 2008.

[3] L. Venkataramanan, Y. Q. Song, and M. D. Hürlimann, "Solving Fredholm integrals of the first kind with tensor product structure in 2 and 2.5 dimensions," IEEE Transactions on Signal Processing, vol. 50, no. 5, pp. 1017-1026, 2002.

[4] J. P. Butler, J. A. Reeds, and S. V. Dawson, "Estimating solutions of first kind integral equations with nonnegative constraints and optimal smoothing," SIAM Journal on Numerical Analysis, vol. 18, no. 3, pp. 381-397, June 1981.

[5] E. Chouzenoux, S. Moussaoui, J. Idier, and F. Mariette, "Efficient maximum entropy reconstruction of nuclear magnetic resonance T1-T2 spectra," IEEE Transactions on Signal Processing, vol. 58, no. 2, pp. 6040-605, December 2010.

[6] F. Mariette, J. P. Guillement, C. Tellier, and P. Marchal, "Continuous relaxation time distribution decomposition by MEM," Signal Treatment and Signal Analysis in NMR, pp. 218-234, 1996.

[7] A. E. English, K. P. Whittall, M. L. G. Joy, and R. M. Henkelman, "Quantitative two-dimensional time correlation relaxometry," Magnetic Resonance in Medecine, vol. 22, pp. 425-434, 1991.

[8] S. Boyd and L. Vandenberghe, Convex Optimization, Cambridge University Press, New York, 1st edition, 2004.

[9] P. Armand, J. C. Gilbert, and S. Jan-Jégou, "A feasible BFGS interior point algorithm for solving strongly convex minimization problems," SIAM Journal on Optimization, vol. 11, pp. 199-222, 2000.

[10] S. G. Nash, "A survey of truncated-Newton methods," Journal of Computational and Applied Mathematics, vol. 124, pp. 4559, 2000.

[11] E. Chouzenoux, S. Moussaoui, and J. Idier, "Majorizeminimize linesearch for inversion methods involving barrier function optimization," Inverse Problems, vol. 28, no. 6, 2012.

[12] A. Conn, N. Gould, and L. Toint, "A primal-dual algorithm for minimizing a nonconvex function subject to bounds and nonlinear constraints," Tech. Rep. RC 20639, Yorktown Heights, NY, 1996, citeseer.ist.psu.edu/ conn96primaldual. html.

[13] A. S. El-Bakry, R. A. Tapia, T. Tsuchiya, and Y. Zhang, "On the formulation and theory of the Newton interior-point method for nonlinear programming," Journal of Optimization Theory and Applications, vol. 89, no. 3, pp. 507-541, 1996. 